\title{
ROBUST STABILITY ANALYSIS OF LINEAR SYSTEMS WITH AFFINE PARAMETER UNCERTAINTIES ${ }^{1}$
}

\author{
Guang-Hong Yang and Kai-Yew Lum
}

\author{
Temasek Laboratories, National University of Singapore, 5 \\ Sports Drive 2, Singapore 117508, Republic of Singapore. \\ Emails: tslygh@nus.edu.sg, tsllumky@nus.edu.sg.
}

\begin{abstract}
This paper is concerned with the problem of robust stability of a class of uncertain linear systems, where the system state-matrices considered are affinely dependent on the uncertain parameters. Affine parameter-dependent Lyapunov functions are exploited to prove stability, and a robust stability criterion for the above class of systems to be affinely quadratically stable (AQS) is given in terms of linear matrix inequalities (LMIs). A comparison with the existing tests for AQS is given, and it is shown that the robust stability criterion provides a test that is not more conservative than the existing tests. Numerical examples are given to illustrate the results. Copyright (C)2005 IFAC
\end{abstract}

Keywords: Linear systems, robust stability, parameter-dependent Lyapunov function, linear matrix inequalities, parameter uncertainty.

\section{INTRODUCTION}

Robust stability analysis for control systems with parameter uncertainties is one of fundamental issues in systems theory, many important advances have been achieved, see (Apkarian et al., 2001; Feron et al., 1996; Gahinet et al., 1996; Scherer, 1997; Tuan and Apkarian, 2002; Zhou et al., 1996) and the references therein. For linear systems with affine parameter uncertainties, a comprehensive development via the notion of quadratic stability (i.e., use of a single quadratic Lyapunov function of the form $V(x)=x^{T} P x$ to prove stability, (Barmish, 1982)) is presented by the Linear Matrix Inequality (LMI) approach in (Boyd et al., 1994). However, the use of a single Lyapunov function for robust stability often leads to overly conservative results. To overcome this, affine parameter-dependent Lyapunov functions

\footnotetext{
1 This work is supported under grant DSTA POD1820, Singapore.
}

which depend on the real uncertain parameters are used to establish robust stability criteria in (Gahinet et al., 1996), which is less conservative than quadratic stability. In (Feron et al., 1996), a sufficient condition for the existence of an affine parameter-dependent Lyapunov function is presented in terms of LMIs, and the resulting stability criterion is less conservative than Popov's stability criterion.

Recently, more general parameter-dependent Lyapunov functions, the Projection Lemma(Gahinet and Apkarian, 1994) and the multiplier technique have been exploited to develop less conservative robust stability criteria (Apkarian et al., 2001; de Oliveira et al., 1999; Dettori and Scherer, 1998; Dettori and Scherer, 2000; Iwasaki, 1997; Iwasaki, 1998; Iwasaki et al., 2001; Peaucelle and Arzelier, 2001; Scherer, 1997). The multiplier technique consists of parameter dependent multipliers (Dettori and Scherer, 2000; Iwasaki, 1998) and parameter independent multipliers (Dettori and 
Scherer, 1998; Iwasaki et al., 2001; Scherer, 1997), which reduces the problems of robust stability and performance analysis to convex optimization problems, and results in various robustness tests. However, the relation among these tests appears to be unclear. Generally, it is difficult to compare the degree of conservatism associated with the proposed robustness tests. But it becomes possible when the type of parameter-dependent Lyapunov functions used to prove stability is specified. Some such comparisons have been done in (Feron et al., 1996; Iwasaki, 1997; Iwasaki et al., 2001)

This paper will address the problem of robust stability analysis for a class of uncertain linear time-invariant systems, where the system state-matrices considered are affinely dependent on the uncertain parameters. Affine parameterdependent Lyapunov functions are exploited to prove stability, and a robust stability criterion for this class of systems to be affinely quadratically stable (AQS)(Gahinet et al., 1996) is given in terms of linear matrix inequalities (LMIs). Comparing with the existing robust stability criteria for AQS, it is shown that the proposed robust stability criterion is no more conservative than the AQS results in (Dettori and Scherer, 2000; Feron et al., 1996; Gahinet et al., 1996; Iwasaki, 1998; Peaucelle and Arzelier, 2001). The paper is organized as follows. The problem statement and some preliminaries are given in Section 2. Section 3 presents the robust stability criterion, and the comparison with the existing robust stability criteria for AQS. Section 4 concludes the paper.

Notation: For a matrix $E, E^{T}$ and $E^{-1}$ denote its transpose and inverse if it exists, respectively. For a column-rank deficient matrix $H, \mathbf{N}_{H}$ denotes a matrix whose columns form a basis for the null space of $H . I$ and $I_{k}$ denote the identity matrix with an appropriate dimension and $k \times k$ identity matrix, respectively.

\section{PROBLEM STATEMENT AND PRELIMINARIES}

Consider an uncertain linear time-invariant system described by

$$
\dot{x}(t)=A(\delta) x(t)
$$

where $x(t) \in R^{n}$ is the state, and $A(\delta)$ is affinely dependent on $\delta$, i.e.,

$$
A(\delta)=A_{0}+\sum_{i=1}^{N} \delta_{i} A_{i}
$$

The real parameter vector $\delta=\left[\delta_{1} \cdots \delta_{N}\right]^{T}$ is uncertain but constant in time and satisfies

$$
\left|\delta_{i}\right| \leq \gamma, \quad i=1, \cdots, N
$$

Definition 1(Feron et al., 1996): The system (1) is said to be affinely quadratically stable (AQS) if there exist $N+1$ symmetric matrices $P_{0}, P_{1}, \cdots, P_{N}$ such that, for all $\delta_{i}$ satisfying (3),

$$
P(\delta):=P_{0}+\sum_{i=1}^{N} \delta_{i} P_{i}>0
$$

and

$$
P(\delta) A(\delta)+A(\delta)^{T} P(\delta)<0
$$

Then the problem considered in this paper is as follows.

Affinely quadratic stability problem: For a given $\gamma>0$, is the system (1) AQS?

Remark 1: For the robust stability problem, many significant advances have been achieved. In (Gahinet et al., 1996), the S-procedure is applied to give a test for AQS, which is less conservative than Popov's stability criterion. Based on the multiconvexity condition for scalar quadratic functions, a different test for AQS is presented in (Feron et al., 1996). Recently, the parameter dependent multiplier approach or the vertex separator approach are exploited to derive robust stability criteria for systems with affine parameter uncertainties in (Dettori and Scherer, 2000) and (Iwasaki, 1998), respectively, and a test for robust stability is derived in (Peaucelle and Arzelier, 2001) by using the Projection Lemma(Gahinet and Apkarian, 1994). All these robust stability criteria also provide tests for AQS. In the next section, we will use the approach in (Iwasaki et al., 2001) to present a sufficient condition for AQS, and then prove that the latter is no more conservative than the above-mentioned ones.

The following preliminaries will be used in the development. The set of vertices of the cube described by (3) is denoted by $\delta_{\mathbf{v}}$, i.e.,

$\delta_{\mathbf{v}}=\left\{\left[\delta_{1} \cdots \delta_{N}\right]^{T}: \delta_{i} \in\{-\gamma, \gamma\}, i=1, \cdots, N\right\}(6)$

Lemma 1 ((Iwasaki et al., 2001; Scherer, 1997)): Let matrices $Q=Q^{T}, F$, and a compact subset of real matrices $\mathbf{H}$ be given. Then the following statements are equivalent:

(i) for each $H \in \mathbf{H}$

$\xi^{T} Q \xi<0$ for all $\xi \neq 0$ such that $H F \xi=0$;

(ii) there exists $\Theta=\Theta^{T}$ such that

$$
Q+F^{T} \Theta F<0, \quad \mathbf{N}_{H}^{T} \Theta \mathbf{N}_{H} \geq 0 \text { for all } H \in \mathbf{H} \text {. }
$$

\section{ROBUST STABILITY ANALYSIS}

Denote

$$
X_{1}=\left[\begin{array}{lll}
x_{1} & \cdots & x_{N}
\end{array}\right]^{T} \in R^{N n}
$$




$$
\begin{aligned}
& E_{1}=\left[\begin{array}{lll}
I_{n} & \cdots & I_{n}
\end{array}\right]^{T} \in R^{N n \times n} \\
& \Delta=\operatorname{diag}\left[\delta_{1} I_{n} \cdots \delta_{N} I_{n}\right] \\
& P^{1}=\left[\begin{array}{lll}
P_{1} & \cdots & P_{N}
\end{array}\right]^{T} \in R^{N n \times n} \\
& A^{1}=\left[\begin{array}{lll}
A_{1} & \cdots & A_{N}
\end{array}\right] \in R^{n \times N n}
\end{aligned}
$$

Then we have

Theorem 1: Consider the uncertain system (1), and assume that $A_{0}$ is Hurwitz. Then the following two statements are equivalent:

(i) the system (1) is affinely quadratically stable;

(ii) there exist symmetric matrices $P_{0}, P_{1}, \cdots, P_{N}$ and $\Theta \in R^{2 N n \times 2 N n}$ such that

$$
\begin{aligned}
& {\left[\begin{array}{l}
P_{0} \\
P^{1}
\end{array}\right]\left[\begin{array}{ll}
A_{0} & A^{1}
\end{array}\right]+\left(\left[\begin{array}{c}
P_{0} \\
P^{1}
\end{array}\right]\left[\begin{array}{ll}
A_{0} & A^{1}
\end{array}\right]\right)^{T}} \\
& +\left[\begin{array}{cc}
E_{1} & 0 \\
0 & I_{N n}
\end{array}\right]^{T} \Theta\left[\begin{array}{cc}
E_{1} & 0 \\
0 & I_{N n}
\end{array}\right]<0
\end{aligned}
$$

and

$$
\left[\begin{array}{l}
I \\
\Delta
\end{array}\right]^{T} \Theta\left[\begin{array}{l}
I \\
\Delta
\end{array}\right] \geq 0 \text { for all } \delta_{i} \text { satisfying (3) }
$$

hold.

Proof: By definition 1, (i) is equivalent to that there exist $N+1$ symmetric matrices $P_{0}, P_{1}, \cdots, P_{N}$ such that (4) and (5) holds for all $\delta_{i}$ satisfying (3). From (7)-(11) and (2), it follows

$$
\begin{gathered}
x^{T} P(\delta) A(\delta) x=\left(x^{T} P_{0}+X_{1}^{T} P^{1}\right)\left(A_{0} x+A^{1} X_{1}\right) \\
=\left[\begin{array}{c}
x \\
X_{1}
\end{array}\right]^{T}\left[\begin{array}{l}
P_{0} \\
P^{1}
\end{array}\right]\left[\begin{array}{ll}
A_{0} & A^{1}
\end{array}\right]\left[\begin{array}{c}
x \\
X_{1}
\end{array}\right] \\
X_{1}=\Delta E_{1} x
\end{gathered}
$$

(15) is equivalent to

$$
\left[\begin{array}{ll}
\Delta & -I
\end{array}\right]\left[\begin{array}{cc}
E_{1} & 0 \\
0 & I_{N n}
\end{array}\right]\left[\begin{array}{c}
x \\
X_{1}
\end{array}\right]=0
$$

By Lemma 1, (14) and (16), the equivalence of (5) and (ii) follows. Furthermore, by the result in (Feron et al., 1996), conditions (4) and (5) are equivalent to (5) under the assumption that $A_{0}$ is Hurwitz. Thus, the proof is complete.

Theorem 1 gives a necessary and sufficient condition for the uncertain system (1) to be AQS via the parameter dependent Lyapunov function $V(x)=x^{T} P(\delta) x$ with $P(\delta)$ given by (4). However, condition (13) is nonconvex, it is difficult to be verified. The following theorem presents a convex sufficient condition.

Theorem 2: The system (1) is affinely quadratically stable if $A_{0}$ is Hurwitz and, if there exist symmetric matrices $P_{0}, P_{i}(i=1, \cdots, N)$, and a symmetric matrix $\Theta$ given by

$$
\Theta=\left[\begin{array}{ll}
\Theta_{11} & \Theta_{12} \\
\Theta_{12}^{T} & \Theta_{22}
\end{array}\right]
$$

with $\Theta_{11} \in R^{N n \times N n}$, and

$$
\Theta_{22 i i} \leq 0, \quad i=1, \cdots, N
$$

such that (12) holds and, moreover,

$$
\left[\begin{array}{l}
I \\
\Delta
\end{array}\right]^{T} \Theta\left[\begin{array}{c}
I \\
\Delta
\end{array}\right] \geq 0 \text { for all } \delta \in \delta_{\mathbf{v}}
$$

where $\Theta_{22 i i} \in R^{s_{p} \times s_{p}}(i=1, \cdots, N)$ are the block matrices on the diagonal of $\Theta_{22}$.

Proof: This follows from Theorem 1, and the method in (Iwasaki et al., 2001). Details are omitted.

Theorem 2 presents a sufficient condition for affinely quadratic stability (AQS) of affine parameterdependent systems, which is from the vertex separator approach or the parameter-independent multiplier approach (Iwasaki et al., 2001; Dettori and Scherer, 1998). The robust stability condition is convex, and can be checked by solving a set of LMIs. For the topic of AQS, there have been some applicable analysis methods (Dettori and Scherer, 2000; Feron et al., 1996; Gahinet et al., 1996; Iwasaki, 1998; Peaucelle and Arzelier, 2001). However, the relation among these tests appears to be unclear. The following theorem will show that the robust stability criterion given in Theorem 2 is no more conservative than the existing ones.

Theorem 3: Consider the uncertain system (1), and $P(\delta)$ given by (4). Assume that $A_{0}$ is Hurwitz. Then the condition in Theorem 2 is feasible, i.e., there exist symmetric matrices $P_{0}, P_{i}(i=$ $1, \cdots, N)$, and a symmetric matrix $\Theta$ given by (17) such that (18), (12) and (19) hold, if one of the following robust stability conditions holds:

(i) there exist symmetric matrices $P_{0}, P_{1}, \cdots, P_{N}$ and $V_{0}$ with $V_{0} \leq 0$, and matrices $S_{1}$ and $S_{2}$ such that

$$
\begin{aligned}
M_{1}(\Delta):= & {\left[\begin{array}{c}
S_{1}^{T} A(\delta)+A(\delta)^{T} S_{1}-A(\delta)^{T} V_{0} A(\delta) \\
P(\delta)-S_{1}^{T}+A(\delta)^{T} S_{2}^{T} \\
P(\delta)-S_{1}^{T}+A(\delta)^{T} \\
\\
-S_{2}-S_{2}^{T}+V_{0}
\end{array}\right]<0, \text { for } \delta \in \delta_{\mathbf{v}}(20) }
\end{aligned}
$$

(ii) there exist symmetric matrices $P_{0}, P_{1}, \cdots, P_{N}$ and $V=\left[V_{i j}\right]_{N \times N} \in R^{N n \times N n}$ with $V_{i i} \in R^{n \times n}$ and

$$
V_{i i} \leq 0, \quad i=1, \cdots, N
$$

and matrices $W$ and $Z$ such that 


$$
\begin{aligned}
M_{2}(\Delta): & =\left[\begin{array}{cc}
P(\delta) A_{0}+A_{0}^{T} P(\delta) & P(\delta) A^{1} \\
\left(A^{1}\right)^{T} P(\delta) & 0
\end{array}\right] \\
& +\left[\begin{array}{cc}
E_{1} & 0 \\
0 & I_{N n}
\end{array}\right]^{T} \Theta(\Delta)\left[\begin{array}{cc}
E_{1} & 0 \\
0 & I_{N n}
\end{array}\right] \\
& <0, \text { for } \delta \in \delta_{\mathbf{v}}
\end{aligned}
$$

where

$$
\Theta(\Delta)=\left[\begin{array}{cc}
\Theta_{11} & W^{T}+\Delta^{T} Z^{T} \\
W+Z \Delta & V-Z-Z^{T}
\end{array}\right]
$$

with $\Theta_{11}=-\left(\Delta^{T} V \Delta+\Delta^{T} W+W^{T} \Delta\right)$.

(iii) there exist symmetric matrices $P_{0}, P_{1}, \cdots, P_{N}$ and $M_{i}, i=1, \cdots, N$ such that

$$
\begin{gathered}
P_{i} A_{i}+A_{i}^{T} P_{i}+M_{i} \geq 0 \text { for } i=1, \cdots, N \\
M_{i} \geq 0 \text { for } i=1, \cdots, N
\end{gathered}
$$

and

$$
\begin{aligned}
M_{3}(\Delta):= & P(\Delta) A(\Delta)+A(\Delta)^{T} P(\Delta) \\
& +\sum_{i=1}^{N} \delta_{i}^{2} M_{i}<0 \text { for all } \delta \in \delta_{\mathbf{v}}
\end{aligned}
$$

(iv) there exist symmetric matrices $P_{0}, P_{1}, \cdots, P_{N}$ and $S=\operatorname{diag}\left[S_{1} \cdots S_{N}\right]$ with $S_{i} \in R^{n \times n}$, and a skew-symmetric matrix $T_{s}=\operatorname{diag}\left[T_{s 1} \cdots T_{s N}\right]$ with $T_{s i} \in R^{n \times n}$ such that $S>0$ and

$$
\begin{aligned}
& {\left[\begin{array}{c}
P_{0} \\
P^{1}
\end{array}\right]\left[\begin{array}{ll}
A_{0} & A^{1}
\end{array}\right]+\left(\left[\begin{array}{l}
P_{0} \\
P^{1}
\end{array}\right]\left[\begin{array}{cc}
A_{0} & A^{1}
\end{array}\right]\right)^{T} } \\
+ & {\left[\begin{array}{cc}
E_{1} & 0 \\
0 & I_{N n}
\end{array}\right]^{T}\left[\begin{array}{cc}
\gamma^{2} S & -T_{s} \\
T_{s} & -S
\end{array}\right]\left[\begin{array}{cc}
E_{1} & 0 \\
0 & I_{N n}
\end{array}\right]<O(27) }
\end{aligned}
$$

The following lemmas will be used in the proof of Theorem 3.

Lemma 2: Let $W \in R^{(N+1) n \times(N+1) n}$ be a symmetric matrix. If

$$
\left[\begin{array}{c}
I \\
F_{1} \\
\vdots \\
F_{N}
\end{array}\right]^{T} W\left[\begin{array}{c}
I \\
F_{1} \\
\vdots \\
F_{N}
\end{array}\right] \geq 0
$$

holds for any $F_{i} \in R^{n \times n}(i=1, \cdots, N)$, then $W \geq 0$.

Proof: If $W \geq 0$ does not hold, then there exists a

$$
\begin{gathered}
\text { vector }\left[\begin{array}{c}
x \\
y_{1} \\
\vdots \\
y_{N}
\end{array}\right] \text { with } x \neq 0 \text { such that } \\
{\left[\begin{array}{c}
x \\
y_{1} \\
\vdots \\
y_{N}
\end{array}\right]^{T} W\left[\begin{array}{c}
x \\
y_{1} \\
\vdots \\
y_{N}
\end{array}\right]<0}
\end{gathered}
$$

Since $x \neq 0$, we have matrices $F_{1}, \cdots, F_{N}$ such that $y_{i}=F_{i} x, i=1, \cdots, N$. Thus,

$$
x^{T}\left[\begin{array}{c}
I \\
F_{1} \\
\vdots \\
F_{N}
\end{array}\right]^{T} W\left[\begin{array}{c}
I \\
F_{1} \\
\vdots \\
F_{N}
\end{array}\right] x<0
$$

which contradicts with (28).

Denote

$$
\begin{gathered}
F=\operatorname{diag}\left[F_{1} \cdots F_{N}\right] \\
P(F)=P_{0}+\sum_{i=1}^{N} F_{i} P_{i} \\
A(F)=A_{0}+\sum_{i=1}^{N} A_{i} F_{i}
\end{gathered}
$$

$$
\begin{aligned}
M_{1 R}(F)= & S_{1}^{T} A(F)+A(F)^{T} S_{1}-A(F)^{T} V_{0} A(F) \\
& +\left(P(\delta)-S_{1}^{T}+A(\delta)^{T} S_{2}^{T}\right) \\
& \times\left(S_{2}+S_{2}^{T}-V_{0}\right)^{-1} \\
& \times\left(P(\delta)-S_{1}^{T}+A(\delta)^{T} S_{2}^{T}\right)^{T}
\end{aligned}
$$

$$
\begin{aligned}
& M_{1 R E}=\left[M_{1 R E i j}\right]_{(N+1) \times(N+1)} \\
& :=\left[\begin{array}{c}
S_{1}^{T}\left[A_{0} A^{1}\right] \\
0
\end{array}\right]+\left[\begin{array}{c}
S_{1}^{T}\left[A_{0} A^{1}\right] \\
0
\end{array}\right]^{T} \\
& -\left[\begin{array}{ll}
A_{0} & A^{1}
\end{array}\right]^{T} V_{0}\left[\begin{array}{ll}
A_{0} & A^{1}
\end{array}\right]+\left(\left[\begin{array}{c}
P_{0} \\
P^{1}
\end{array}\right]-\left[\begin{array}{c}
S_{1}^{T} \\
0
\end{array}\right]\right. \\
& \left.+\left[A_{0} A^{1}\right]^{T} S_{2}^{T}\right)\left(S_{2}+S_{2}^{T}-V_{0}\right)^{-1} \\
& \times\left(\left[\begin{array}{c}
P_{0} \\
P^{1}
\end{array}\right]-\left[\begin{array}{c}
S_{1}^{T} \\
0
\end{array}\right]+\left[\begin{array}{ll}
A_{0} & A^{1}
\end{array}\right]^{T} S_{2}^{T}\right)
\end{aligned}
$$

with $M_{1 R E i i} \in R^{n \times n}(i=0,1, \cdots, N)$.

$$
\begin{aligned}
& M_{2 R}(F)=P(F) A_{0}+A_{0}^{T} P(F)^{T} \\
& -\left(E_{1}^{T} F^{T} V F E_{1}+E_{1}^{T} F^{T} W+W^{T} F E_{1}\right) \\
& +\left(P(F) A^{1}+W^{T}+E_{1}^{T} F^{T} Z^{T}\right)\left(Z+Z^{T}-V\right)^{-1} \\
& \times\left(P(F) A^{1}+W^{T}+E_{1}^{T} F^{T} Z^{T}\right)^{T} \\
& M_{2 R E}=\left[M_{2 R E i j}\right]_{(N+1) \times(N+1)} \\
& :=\left[\left[\begin{array}{c}
P_{0} \\
P^{1}
\end{array}\right] A_{0} \quad 0 \quad \cdots \quad 0\right] \\
& +\left[\left[\begin{array}{l}
P_{0} \\
P^{1}
\end{array}\right] A_{0} \quad 0 \quad \cdots \quad\right]^{T} \\
& -\operatorname{diag}[0 \quad V]-\left[\begin{array}{cc}
0 & W \\
0 & 0
\end{array}\right]-\left[\begin{array}{cc}
0 & W \\
0 & 0
\end{array}\right]^{T} \\
& +\left(\left[\begin{array}{c}
P_{0} \\
P^{1}
\end{array}\right] A^{1}+\left[\begin{array}{c}
W^{T} \\
0
\end{array}\right]+\left[\begin{array}{c}
0 \\
Z^{T}
\end{array}\right]\right) \\
& \times\left(Z+Z^{T}-V\right)^{-1} \\
& \times\left(\left[\begin{array}{c}
P_{0} \\
P^{1}
\end{array}\right] A^{1}+\left[\begin{array}{c}
W^{T} \\
0
\end{array}\right]+\left[\begin{array}{c}
0 \\
Z^{T}
\end{array}\right]\right)^{T}(35)
\end{aligned}
$$


with $M_{2 R E i i} \in R^{n \times n}(i=0,1, \cdots, N)$.

$$
\begin{aligned}
M_{3 R E}= & {\left[M_{2 R E i j}\right]_{(N+1) \times(N+1)} } \\
:= & {\left[\begin{array}{l}
P_{0} \\
P^{1}
\end{array}\right]\left[\begin{array}{ll}
A_{0} & A^{1}
\end{array}\right]+\left(\left[\begin{array}{l}
P_{0} \\
P^{1}
\end{array}\right]\left[\begin{array}{ll}
A_{0} & A^{1}
\end{array}\right]\right)^{T} } \\
& +\operatorname{diag}\left[0 M_{11} \cdots M_{N N}\right]
\end{aligned}
$$

Lemma 3: (i) If $S_{2}+S_{2}^{T}-V_{0}>0$, then

$$
\left[\begin{array}{l}
P_{0} \\
P^{1}
\end{array}\right]\left[A_{0} A^{1}\right]+\left(\left[\begin{array}{c}
P_{0} \\
P^{1}
\end{array}\right]\left[\begin{array}{ll}
A_{0} & A^{1}
\end{array}\right]\right)^{T} \leq M_{1 R E}
$$

(ii) If $Z+Z^{T}-V>0$, then

$$
\left[\begin{array}{l}
P_{0} \\
P^{1}
\end{array}\right]\left[\begin{array}{ll}
A_{0} & A^{1}
\end{array}\right]+\left(\left[\begin{array}{c}
P_{0} \\
P^{1}
\end{array}\right]\left[\begin{array}{ll}
A_{0} & A^{1}
\end{array}\right)^{T} \leq M_{2 R E}\right.
$$

(iii) If $M_{i i} \geq 0(i=1, \cdots, N)$, then

$$
\left[\begin{array}{l}
P_{0} \\
P^{1}
\end{array}\right]\left[A_{0} A^{1}\right]+\left(\left[\begin{array}{c}
P_{0} \\
P^{1}
\end{array}\right]\left[\begin{array}{ll}
A_{0} & A^{1}
\end{array}\right)^{T} \leq M_{3 R E}\right.
$$

Proof: It is omitted here, due to the space limitation.

Proof of Theorem 3: (i) By Schur complement and (32), (20) is equivalent to that $S_{2}+S_{2}^{T}-V_{0}>0$ and

$$
M_{1 R}(\Delta)<0
$$

By Lemma 1 and (??), it follows that there exists a symmetric matrix $\Theta=\left[\begin{array}{cc}\Theta_{11} & \Theta_{12} \\ \Theta_{12}^{T} & \Theta_{22}\end{array}\right]$ such that

$$
M_{1 R E}+\left[\begin{array}{cc}
E_{1} & 0 \\
0 & I_{N n}
\end{array}\right]^{T} \Theta\left[\begin{array}{cc}
E_{1} & 0 \\
0 & I_{N n}
\end{array}\right]<0
$$

and (13) hold. From (33), $V_{0} \leq 0$ and $S_{2}+S_{2}^{T}-$ $V_{0}>0$, it follows that

$$
M_{1 R i i} \geq 0, \quad i=1, \cdots, N
$$

which further implies that $\Theta_{22 i i} \leq 0, \quad i=$ $1, \cdots, N$. Thus, from Lemma 2, (19) holds and (12) follows from (37).

(ii) By Schur complement and (34), it follows that (22) is equivalent to

$$
M_{2 R}(\Delta)<0
$$

The rest of the proof can be completed by (38), (21), (42) and the arguments similar to those for (i), and the details are omitted.

(iii) By (39) and $M_{i i} \geq 0(i=1, \cdots, N)$, it is immediate.

(iv) Notice that $\Theta=\left[\begin{array}{cc}\gamma^{2} S & -T_{s} \\ T_{s} & -S\end{array}\right]$ satisfies (18) and (19), so the conclusion follows.

Remark 2: Theorem 3 presents a comparison between Theorem 2 and the existing results for
AQS, where $P(\delta)$ is required to be in the form of (4). Condition (i) is an extension of the results for AQS in (Dettori and Scherer, 2000) and (Peaucelle and Arzelier, 2001), where (Peaucelle and Arzelier, 2001) applies the Projection Lemma (Gahinet and Apkarian, 1994) to give the following condition for AQS: $A_{0}$ is Hurwitz, and there exist symmetric matrices $P_{0}, P_{1}, \cdots, P_{N}$, and matrices $S_{1}$ and $S_{2}$ such that

$$
\begin{aligned}
& M_{1}(\Delta):=\left[\begin{array}{c}
S_{1}^{T} A(\delta)+A(\delta)^{T} S_{1} \\
\left(P(\delta)-S_{1}^{T}+A(\delta)^{T} S_{2}^{T}\right)^{T}
\end{array}\right. \\
& \left.\begin{array}{c}
P(\delta)-S_{1}^{T}+A(\delta)^{T} S_{2}^{T} \\
-S_{2}-S_{2}^{T}
\end{array}\right] \\
& <0 \text {, for } \delta \in \delta_{\mathbf{v}}
\end{aligned}
$$

which is same as the result in (Dettori and Scherer, 2000), and corresponds to a special case of condition (i) when $V_{0}=0$. Condition (ii) is given in (Iwasaki, 1998). The stability criteria in (Dettori and Scherer, 2000) and (Iwasaki, 1998) are obtained by using parameter-dependent multiplier or separator approach. Condition (iii) is from (Gahinet et al., 1996), which is based on the result on multiconvexity(Iwasaki et al., 2001). Condition (iv) is given in (Feron et al., 1996), which is derived by using S-procedure approach. Theorem 3 shows that the stability criterion given by using vertex separator approach or parameterindependent multiplier approach(Iwasaki et al., 2001; Dettori and Scherer, 1998) provides a stability test that is no more conservative than the above existing results for AQS.

Example 1: The considered uncertain linear time-invariant system is described by (1)-(3) with

$$
\begin{gathered}
A_{0}=\left[\begin{array}{ccc}
-5 & 2 & 1 \\
-10 & -5 & -1 \\
5 & 1 & -6
\end{array}\right], \quad A_{1}=\left[\begin{array}{lll}
0 & 1 & 0 \\
0 & 0 & 0 \\
0 & 0 & 0
\end{array}\right] \\
A_{2}=\left[\begin{array}{lll}
0 & 0 & 0 \\
0 & 0 & 0 \\
0 & 1 & 0
\end{array}\right], \quad A_{3}=\left[\begin{array}{lll}
0 & 0 & 0 \\
0 & 1 & 1 \\
0 & 0 & 1
\end{array}\right]
\end{gathered}
$$

We are interested in computing the maximal value of $\gamma$ such that the system is AQS. The optimal $\gamma$ for each method is given in Table 1. It is as expected that Theorem 2 gives the best stability margin $\gamma=2.957$ for the system to be AQS. However, it requires more computational time than most of the existing tests.

Regarding computational complexity, the number of variables and the number of LMIs are used as measures of computational burden. It is easy to see that the methods given in (Iwasaki, 1998) and Theorem 2 require more variables than others, and the methods in (Dettori and Scherer, 2000), (Peaucelle and Arzelier, 2001), (Iwasaki, 1998), (Gahinet et al., 1996), Condition (i) in Theorem 3 and Theorem 2 involve conditions on the vertices 
of the parameter set and require exponentially growing numbers of LMI constraints with respect to $N$. So the methods given in (Iwasaki, 1998) and Theorem 2 will be computationally very demanding, while the method in (Feron et al., 1996) is of less computational burden than others when $n$ and $N$ are large. In Table $1, \mathrm{DS}$ and PA represent the methods in (Dettori and Scherer, 2000) and (Peaucelle and Arzelier, 2001), respectively.

Table 1. Optimal $\gamma$ for each method

\begin{tabular}{|l|c|}
\hline Method & $\gamma$ \\
\hline \hline Theorem 2 & 2.957 \\
\hline Condition (i) in Theorem 3 & 2.943 \\
\hline DS, PA & 2.943 \\
\hline (Iwasaki, 1998) & 2.957 \\
\hline (Gahinet et al., 1996) & 2.936 \\
\hline (Feron et al., 1996) & 1.38 \\
\hline
\end{tabular}

Remark 3: It should be pointed out that the tests for AQS given by Condition (i) in Theorem 3, (Dettori and Scherer, 2000), (Peaucelle and Arzelier, 2001) and (Iwasaki, 1998) (see (43) and (ii) in Theorem 3) are also applicable for the case where polytopic Lyapunov functions are used to prove robust stability, which is a more general type of Lyapunov functions than that affine parameterdependent Lyapunov functions considered here.

Remarks 4: In (Tuan and Apkarian, 2002), based on monotonicity concept, a sufficient condition for AQS is given in terms of LMIs. For the uncertain system in Example 1, the optimal $\gamma$ for the method in (Tuan and Apkarian, 2002) is 2.927, which shows that the method is more conservative than Theorem 2 for this numerical example. However, it is difficult to prove the relationship between Theorem 2 and the method in (Tuan and Apkarian, 2002) for the general case.

\section{CONCLUSIONS}

In this paper, we have investigated the problem of affinely quadratic stability (AQS) of a class of uncertain linear systems, where the system statematrices considered are affinely dependent on the uncertain parameters, and affine parameterdependent Lyapunov functions are exploited to prove stability. A robust stability criterion for the class of systems to be affinely quadratically stable (AQS) is given in terms of linear matrix inequalities (LMIs), which is based on the vertex separator approach or parameter-independent multiplier approach(Iwasaki et al., 2001; Dettori and Scherer, 1998). The comparison with the existing tests for AQS is given, and it is shown that the robust stability criterion is no more conservative than the existing tests. A further research topic is to extend the comparison to cases where more general types of parameter-dependent Lyapunov functions such as polytopic Lyapunov functions, and Lyapunov functions that depend on the parameters in a linear fractional manner (Iwasaki et al., 2001). Obviously, such an extension would be very significant, but nontrivial.

\section{REFERENCES}

Apkarian, P., H.D. Tuan and J. Bernussou (2001). IEEE Trans. on Automatic Control 46, 19411946.

Barmish, B.R. (1982). IEEE Trans. on Automatic Control AC-27, 848-850.

Boyd, S., L. El Ghaoui, E. Feron and V. Balakrishan (1994). Linear Matrix Inequalities in Systems and Control Theory. SIAM. Philadelphia.

de Oliveira, M.C., J. Bernussou and J.C. Geromel (1999). Systems \& Control Letters 37, 261265.

Dettori, M. and C.W. Scherer (1998). Proc. 37th IEEE Conf. on Decision and Control, Tampa, Florida pp. 2798-2799.

Dettori, M. and C.W. Scherer (2000). Proc. 39th IEEE Conf. on Decision and Control, Sydney, Australia pp. 4187-4192.

Feron, E., P. Apkarian and P. Gahinet (1996). IEEE Trans. on Automatic Control AC41, 1041-1046.

Gahinet, P. and P. Apkarian (1994). Int. J. Robust Nonlinear Control 4, 421-448.

Gahinet, P., P. Apkarian and M. Chilali (1996). IEEE Trans. on Automatic Control AC41, 436-442.

Iwasaki, T. (1997). Proc. 36th IEEE Conf. on Decision and Control, San Diego, California AC-41, 4880-4885.

Iwasaki, T. (1998). Proc. 37th IEEE Conf. on Decision and Control, Tampa, Florida AC41, 3021-3026.

Iwasaki, T., and G. Shibata (2001). IEEE Transactions on Automatic Control 46, 1195-1208.

Peaucelle, D. and D. Arzelier (2001). IEEE Transactions on Automatic Control 46, 624-630.

Scherer, C.W. (1997). Proc. 36th IEEE Conf. on Decision and Control, San Diego, CA pp. 2602-2607.

Tuan, H.D. and P. Apkarian (2002). IEEE Transactions on Automatic Control 47, 378-384.

Zhou, K., J.C. Doyle and K. Glover (1996). Robust and Optimal Control. Prentice Hall. New Jersey. 